International Journal of Computer Networks \& Communications (IJCNC) Vol.3, No.5, Sep 2011

\title{
Reliable Multicast MAC Protocol For SPATIAL REUSE EFFICIENCY ASSISTED BY MULTICARRIER DS-CDMA IN WLAN SYSTEMS
}

\author{
Jeonghun Kim, Junwoo Jung and Jaesung Lim \\ Mobile Communication Laboratory, Ajou University, Suwon City, Korea \\ kuraterry@hanmail.net, junu000@ajou.ac.kr, jaslimeajou.ac.kr
}

\begin{abstract}
We propose a reliable multicast MAC protocol based on the multicarrier direct-sequence code-division multiple access (MC DS-CDMA) scheme to enhance spatial reuse efficiency in wireless local area networks (WLANs). Conventional multicast MAC protocols using RTS-CTS handshake exchange CTS and ACK packets consecutively for reliable transmission. Although this protocol guarantees transmission reliability by avoiding the hidden node $(H N)$ problem, multiple CTSs and ACKs degrade network efficiency and cause the well-known exposed node (EN) problem, rather than the HN problem. The multicast sender must receive multiple CTSs and ACKs from all multicast receivers in its own multicast group for reliable multicast. The range of the EN problem is greater than that of the unicast, and spatial reuse efficiency decreases. In the proposed protocol, CTS/ACK messages of multiple receivers can be transmitted concurrently through the MC DS-CDMA mechanism to alleviate the EN problem and enhance spatial reuse efficiency. This can reduce the transmission and carrier sensing range of multiple receivers, since the protocol spreads the CTS/ACK transmission power by the spreading gain. The proposed protocol also reduces the total transmission delay due to the reduction of the overhead of consecutive CTSs and ACKs. The proposed protocol is thoroughly examined by simulation and theoretical methods. Results show that the proposed protocol significantly reduces the aforementioned overhead, and thus improves the performance of WLAN systems.
\end{abstract}

\section{KEYWORDS}

MC DS-CDMA, Multicast MAC, Reliability, Exposed Node, Spatial Reuse, Wireless LAN

\section{INTRODUCTION}

Various multicast schemes have recently been introduced into wireless communication systems to improve channel efficiency. The multicast technique achieves better channel efficiency in transmitting the same packets to receivers than the unicast method [1]. Unlike broadcast, whose transmission targets all devices within radio range, multicast targets devices belonging to a particular receiver group. Several issues, including reliable multicast MAC protocol design, challenge the design of a multicast MAC protocol.

IEEE 802.11 standards specify multicast services, as well as unicast services in layer 2 . However, most parts of the protocol specifications are limited to the unicast environment, and multicast has mainly been studied to focus on the relatively higher layers, such as the network and transport layers, as seen in [2-3].

Research on MAC layer multicasting has mainly been based on IEEE 802.11 WLAN and mobile ad-hoc networks. Several MAC layer multicast protocols [4-7] have been introduced. However, they have some problems including MAC overhead and reliability. In [4-6], reliable multicast protocols using multiple CTSs and ACKs were proposed. However, they still have the

DOI : 10.5121/ijcnc.2011.3509 
International Journal of Computer Networks \& Communications (IJCNC) Vol.3, No.5, Sep 2011

overhead problem due to the cumulative transmission of CTSs and ACKs. Although the protocol proposed in [7] concentrated on reducing the overhead, this does not support reliable service. Until now, there was a tradeoff between guaranteeing reliability and reducing overhead in multicast MAC protocols.

Furthermore, there are well-known problems, such as $\mathrm{HN}$ and EN, in multicast services based on IEEE 802.11 standards. With the RTS-CTS handshake, the HN problem can nearly be resolved. However EN caused by carrier sensing of the neighbor node, whose transmission is unrelated, cannot be solved. Furthermore the carrier sensing range, surrounding the multiple receivers, drastically increases in a multicast environment. This defers transmissions of other multicast groups, so the total network efficiency decreases by the EN problem.

In this paper, we propose a new reliable multicast MAC protocol assisted by MC DS-CDMA, to resolve the EN problem, as well as the multicast MAC overhead and reliability. When multiple receivers receive the RTS/DATA message from a sender, each receiver sends a CTS/ACK message spread with a given MC DS-CDMA code. This MAC protocol can reduce the MAC overhead of multiple CTSs/ACKs via concurrent transmissions. If the power control is enabled, both transmission and carrier sensing range of multiple receivers, through the processing gain of the allocated code, can be reduced. However, the codelength of MC DS-CDMA is a tradeoff. If the codelength is enlarged, the carrier sensing range is smaller, i.e. the effect of the EN problem lessens, while the CTS/ACK time is longer. The proposed protocol is thoroughly examined by simulation and analytical methods. The results show that it significantly reduces the aforementioned overhead, and thus improves the performance of the WLAN system.

The remainder of the paper is structured as follows. Section 2 reviews previous wireless multicast research. In section 3, the proposed protocol is detailed. In section 4 and 5 , we evaluate the proposed protocol with mathematical and simulation methods, respectively. Finally the conclusion is given in section 6 .

\section{RELATED WORKS}

\subsection{Multicast MAC Protocol based on IEEE 802.11}

Multicast aware MAC protocol (MMP) [5] and a multiple access collision avoidance protocol for multicast services [6] were proposed for reliable multicast. These kinds of protocol use multiple CTSs and ACKs sent by each receiver for reliable transmission, as shown in Fig. 1(a). This can be reliable. However, it increases overhead caused by the reception time of multiple CTSs and ACKs. Leader-based protocol (LBP) was proposed for multicast to reduce the overhead caused by multiple CTSs and ACKs [7]. In this work, when a sender transmits a packet, it selects a leader station among the multicast group member stations. Then, only the leader responds with the CTS and ACK corresponding to the RTS/DATA message from the sender. If any member station receives the packet with an error, it transmits a NACK message to the sender. This NACK causes a collision with the ACK of the leader station. If there is a collision after transmission of the data packet, the sender recognizes that at least one station failed to receive the data packet. In this case, it sends the data packet again. LBP, as shown in Fig. 1(b), can reduce overhead. However, when one of the member stations fails to demodulate the multicast RTS, the leader CTS, or the MAC header of a multicast data packet, it cannot send a NACK packet, since it cannot recognize if the received packet is multicast and if it is the destination of the packet. Since the station does not send a NACK, no collision is experienced at the sender. Therefore the LBP may not be reliable in terms of the detection of failed transmission. 
International Journal of Computer Networks \& Communications (IJCNC) Vol.3, No.5, Sep 2011

\begin{tabular}{|c|c|c|c|c|c|c|c|c|c|c|}
\hline Sender & $\left.\operatorname{mRTS}^{\text {SIF }}\right|^{\ominus}$ & sif & s! & & $\stackrel{\text { sif }}{\theta}$ & DATA & $\begin{array}{l}=S \\
\theta\end{array}$ & ${ }_{\theta}^{S}$ & & $\|^{\prime I F S}$ \\
\hline Receiver \#1 & & CTS\#1 & & & & & ACK\#1 & & & \\
\hline Receiver \#2 & & & CTS\#2 & & & & & ACK\#2 & & \\
\hline Receiver \#N & & & & ...... & CTS\#N & & & & ..... & ACK\#N \\
\hline
\end{tabular}

(a)

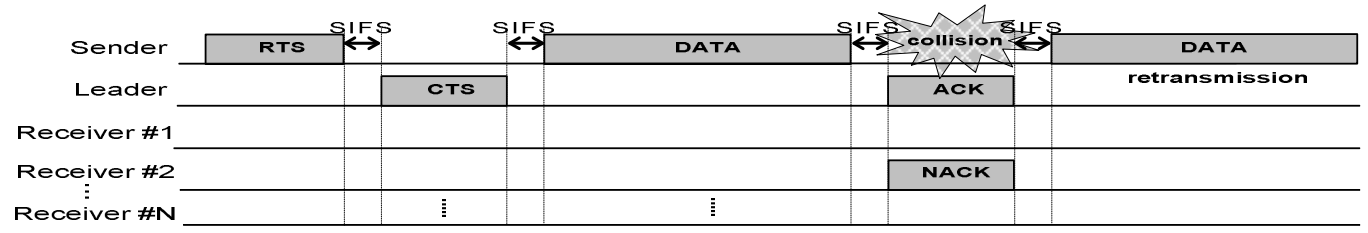

(b)

Figure 1. Example scenarios of (a) Reliable multicast with multiple CTSs and ACKs and (b) LBP (Leader Based Protocol)

Orthogonal frequency division multiplex (OFDM) based multicast ACK (OMACK) was proposed to reduce MAC overhead and to guarantee reliability [8]. This protocol uses one OFDM symbol for ACKs from all member stations. Each member station indicates its packet reception status by utilizing a sub-carrier within the OFDM symbol. This can reduce MAC overhead. However, this can only be adopted for small-sized packet transmissions, since this does not use the RTS-CTS handshake, and cannot solve the spatial reuse problem of EN, explained later.

Until now, the research on the multicast MAC protocol based on IEEE 802.11 standards has been conducted to reduce overhead or to guarantee reliability. There is a tradeoff between the overhead reduction and guaranteeing reliability. If one side is improved, the other side is worsened.

\subsection{EN problem in Multicast Mechanism}

The HN and EN problems still remain in a multicast environment, such as in a unicast one. Considering the RTS-CTS handshake, the HN can almost be solved, but the EN cannot be solved by this.

The EN problem may occur as follows in a unicast environment, as shown in Fig. 2(a). When neighboring nodes, not related to the original transmission, overhear the transmission of other multicast groups, they set the network allocation vector (NAV) to prevent collision between multicast groups. In a multicast environment, as shown in Fig. 2(b), $\mathrm{C} 1$ is in the carrier sensing range of $\mathrm{B} 1$ and $\mathrm{B} 2$. Then multicast sender D sends an RTS message to $\mathrm{C} 1-4$ member stations. However C1 cannot respond with CTS, because it sets the NAV. The multicast transmission between D and C1-4 multicast group is deferred, though it has no influence on A and B1-6 multicast group transmission. Finally $\mathrm{C} 1$ is the EN. The EN range, as shown in Fig. 2(b), in the multicast is expanded more than that in the unicast. Considering multiple multicast groups in a network, EN prevents concurrent transmissions of each multicast group. In conclusion, the network efficiency is severely degraded. Until now, research on EN mainly dealt with the unicast environment [9-11]. Some research proposed solutions for RTS overhearing range or CTS overhearing range. However, the CTS overhearing range is relatively much larger than the 
RTS overhearing range in a multicast environment, as shown in Fig. 2(b) Therefore, we propose a solution for the CTS overhearing range.

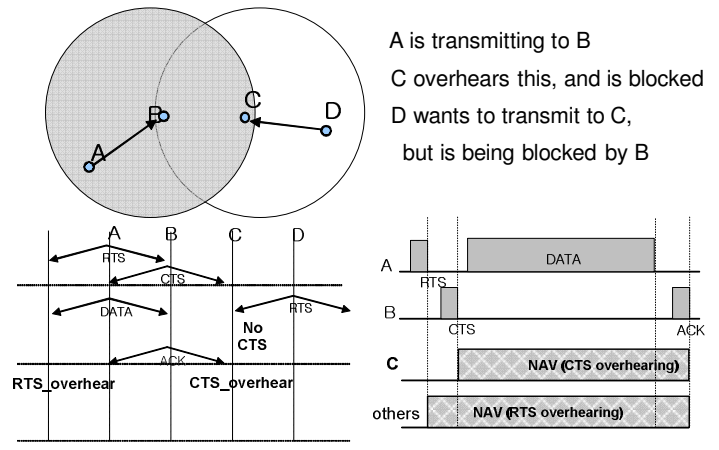

(a)

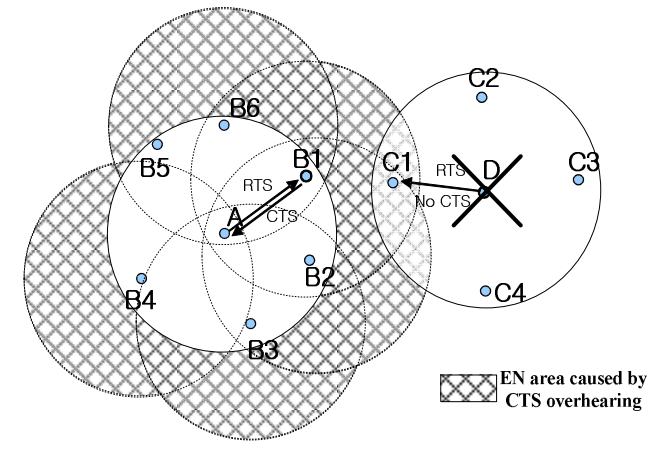

(b)

Figure 2. EN problem in (a) unicast and (b) multicast

\section{Proposed MAC Protocol}

The previous section mentioned the multicast MAC problem that we want to solve. In this section, we propose a new multicast MAC protocol, to solve the EN problem, as well as the multicast MAC overhead, while guaranteeing reliability, assisted by the MC DS-CDMA scheme.

In the proposed protocol, CTS/ACK messages of multiple receivers can be transmitted concurrently through the MC DS-CDMA mechanism to alleviate the EN problem and enhance spatial reuse efficiency.

We use MC DS-CDMA instead of DS-CDMA and MC-CDMA as MC DS-CDMA is of special interest for the quasi-synchronized uplink system, due to its close relationship to the asynchronous single-carrier DS-CDMA system. In our proposal, the reliable multicasting mechanism is similar to uplink transmission, in which multiple receivers respond to one transmitter. Thus, MC DS-CDMA is more attractive than MC-CDMA and single carrier DSCDMA due to its robustness against quasi-synchronization channel [12]. We use MC DSCDMA as the multiple access mechanism.

Let us assume the chip synchronization of the allocated codes coincide with each other for the successful reception of CTS/ACK messages, and each station is able to control the power of the transmission, as the processing gain varies. In addition, when each station responds to the RTS/DATA message, there is a busy tone signal transmission to prevent the HN problem. Finally, the set of spreading codes used in this protocol has MAI-free characteristics.

The mechanism of the proposed multicast MAC protocol is as follows. When multiple receivers receive a RTS/DATA message from a sender, each receiver spreads a CTS/ACK message with the allocated code based on MC-DS/CDMA. Each code allocated to multiple receivers is given by the multicast RTS (mRTS) message from the sender. After waiting for the SIFS time, each receiver sends the CTS/ACK message concurrently and also transmits a busy tone signal. The function of the busy tone signal is explained later in this section. At this time, each receiver can reduce the transmission power level through the processing gain of the MC DS-CDMA codes.

The specific feature of the proposed protocol compared to conventional protocol using multiple CTSs/ACKs is as follows. First, the proposed MAC protocol can reduce the overhead of multiple CTSs and ACKs through concurrent transmissions, as shown in Fig 3(a). However, a 
International Journal of Computer Networks \& Communications (IJCNC) Vol.3, No.5, Sep 2011

single CTS/ACK time spread with the given code is longer than the previous ones. Additionally, the number of multicast group members is restricted by the codelength. If the number of multicast group members exceeds the supportable number, the CTS/ACK is sent at least twice.

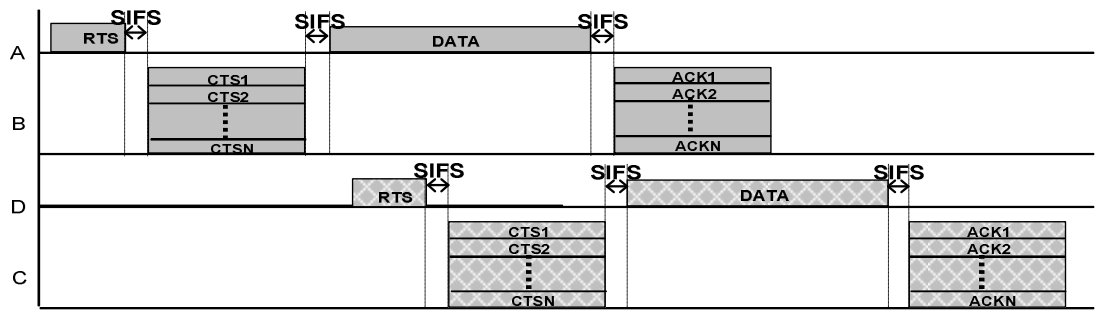

(a)

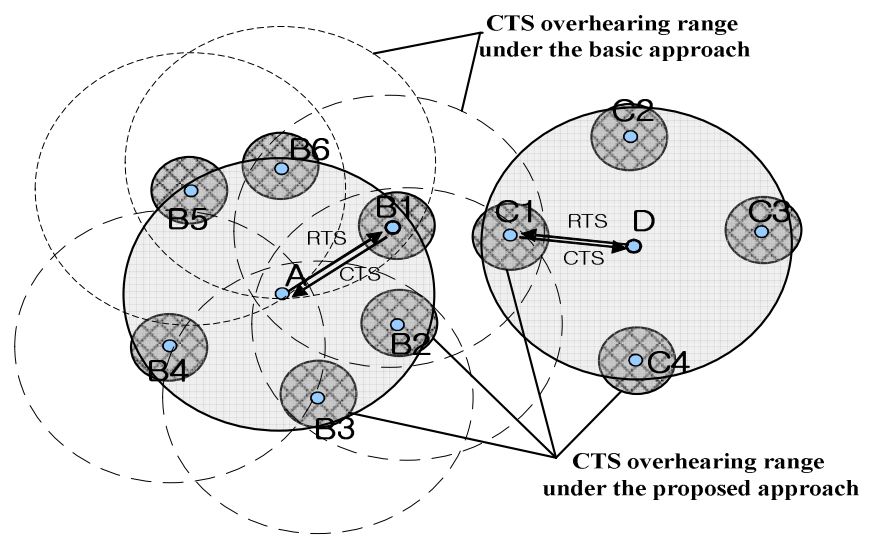

(b)

Figure 3. (a) Proposed MAC Protocol (b) Mitigating EN area assisted by MC DS-CDMA.

Second, the protocol can reduce both the transmission and carrier sensing range of multiple receivers through the processing gain of the codes. Therefore, EN can be reduced. In Fig. 3(b), the multicast group-B members use the proposed protocol. The transmission range and carrier sensing range of multicast receivers are a lot smaller than those in Fig. 2(b). Therefore, since C1 is free from the transmission between multicast group A-B, C1 can respond with CTS corresponding to the RTS message of the sender D. In contrast to $\mathrm{C} 1$, A can decode the CTS/ACK message of multicast group B with the pre-assigned code. In conclusion, a multicast group between $\mathrm{D}$ and $\mathrm{C} 1-\mathrm{C} 4$ can transmit multicast packets concurrently with the multicast group between A and B1-B6.

At this time, if $\mathrm{C} 1$ wants to send a multicast packet, $\mathrm{C} 1$ may be a $\mathrm{HN}$ against $\mathrm{B} 1$ and $\mathrm{B} 2$. To prevent the $\mathrm{HN}, \mathrm{Cl}$ does not have to be a sender. It can only reply with CTS/ACK corresponding to a RTS/DATA message from another sender, such as D. We use the busy-tone solution similar to [10] to solve this problem. When multicast receivers send a CTS message, they divide the busy tone channel and send the busy tone signal until the transmission expires. If a station, such as $\mathrm{C} 1$ in Fig. 3(b), receives a busy-tone signal without receiving a CTS message, it can only be a receiver of another multicast group, not a sender. 
International Journal of Computer Networks \& Communications (IJCNC) Vol.3, No.5, Sep 2011

In conclusion, as the transmission power of receivers decreases, the transmission and carrier sensing range become smaller. Therefore, the EN range reduces. As the EN reduces, the spatial reuse efficiency improves. Finally, the total network efficiency increases. Then, the codelength of MC DS-CDMA becomes a tradeoff. If the codelength is enlarged, the carrier sensing range decreases, i.e. the effect of the EN problem becomes smaller, while the CTS/ACK time lengthens.

In the following section, we will show the margin between the multiple consecutive CTS time and the proposed spread CTS time and find the appropriate codelength, as the number of multicast group members is varied.

The contributions of the proposed multicast MAC protocol are as follows. First, the proposed protocol can reduce the MAC overhead while guaranteeing reliability. Second, we can increase the channel efficiency, as we mitigate the EN problem and increase the spatial reuse ratio (SRR) in the multicast MAC.

\section{Performance Evaluation}

\subsection{Throughput Analysis}

If the network has multiple multicast groups, we have to consider the probability of how many neighboring multicast groups can communicate concurrently. To calculate the total network throughput,

$$
S_{F}=S_{l} \times S R R
$$

where $S_{F}$ refers to the total network throughput. $S_{I}$ is one multicast group's (one sender and multiple receivers) throughput and $S R R$ is the degree of concurrent transmissions in a network. $S R R$ has the value of $1 / N_{G}+1 \sim 1$ (where $N_{G}$, the number of neighbor multicast groups). The notation of $1 / N_{G}+1$ denotes, if there is one transmission in a network, the neighbor groups cannot transmit by EN. $S R R=1$ denotes all neighbor multicast groups can transmit the multicast message independently.

An analytical model is suggested for throughput calculation under saturation. That is, each station has a packet immediately available for transmission [13]. This work models the MAC events of successful transmission, collision and idle waiting. The duration between transitions to each state is called a "slot" and the probability of a slot containing each event is found. For the model in [13] to be correct, two fundamental assumptions are made. First, after each idle slot, each station may attempt to transmit with an independent and constant probability $\tau$. Second, regardless of the number of past collisions, a transmission attempt may result in a collision with an independent and constant probability $p$. The backoff process is then modeled by a twodimensional Markov chain. The following two equations are found for $\tau$ and $p$.

$$
\begin{gathered}
\tau=\frac{2(1-2 p)}{(1-2 p)(W+1)+p W\left(1-(2 p)^{m}\right)} \\
p=1-(1-\tau)^{n-1}
\end{gathered}
$$


International Journal of Computer Networks \& Communications (IJCNC) Vol.3, No.5, Sep 2011

where $n$ is the number of contending stations, $W=C W_{\min }$, Let $m$, "maximum backoff stage", be the value, such that $C W_{\max }=2^{m} W$, and let us adopt the notation $W_{i}=2^{i} W$, where $i \in(0, m)$ is termed the "backoff stage". Solving these equations using numerical methods, one can find $\tau$ and $p$ based on the known values of $W, m$, and $n$.

Let $P_{T}$ be the probability at least one transmission exists in the slot time considered. Since $n$ stations contend on the channel, and each transmits with probability $\tau$.

$$
P_{T}=1-(1-\tau)^{n}
$$

The probability $P_{s}$ that transmission occurring in the channel is successful, is given by the probability that exactly one station transmits in the channel, conditioned on the fact that at least one station transmits, i.e.,

$$
P_{S}=\frac{n \tau(1-\tau)^{n-1}}{P_{T}}=\frac{n \tau(1-\tau)^{n-1}}{1-(1-\tau)^{n}}
$$

We now express $S_{I}$ as the ratio

$$
S_{I}=\frac{E[\mathrm{P}]}{E[s l o t]}
$$

where, $E[$ slot $]$ denotes the length of a slot time. As $E[P]$ is the average packet payload size, the average amount of payload information successfully transmitted in a slot time is $P_{T} P_{S} E[P]$, since a successful transmission occurs in a slot time with probability $P_{T} P_{S}$. E[slot $]$ is readily obtained considering that, with probability $1-\mathrm{P}_{T}$, the slot time is empty; with probability $P_{T} P_{S}$ it contains a successful transmission, and with probability $P_{T}\left(1-P_{S}\right)$, it contains a collision. Hence, (6) becomes

$$
S_{I}=\frac{P_{T} P_{S} E[P]}{\left(1-P_{T}\right) \sigma+P_{T} P_{S} T_{S}+P_{T}\left(1-P_{S}\right) T_{C}}
$$

Here, $T_{S}$ is the average time the channel is sensed busy (i.e., the slot time lasts) due to a successful transmission, and $T_{C}$ is the average time the channel is sensed busy by each station during a collision. $\sigma$ is the duration of an empty slot time.

In a conventional multiple CTS/ACK system of [5] or [6], we can express $T_{S}$ and $T_{C}$ as follow.

$$
\begin{gathered}
T_{S}^{\text {bas }}=R T S+n(S I F S+\delta+C T S)+S I F S+\delta+H+E[P]+n(S I F S+\delta+A C K)+D I F S+\delta \\
T_{C}^{\text {bas }}=R T S+D I F S+\delta
\end{gathered}
$$


International Journal of Computer Networks \& Communications (IJCNC) Vol.3, No.5, Sep 2011

where, let $H=P H Y_{h d r}+M A C_{h d r}$ be the packet header, and $\delta$ be the propagation delay.

In the proposed scheme, the CTS and ACK time is longer than before by the spreading code.

$$
\begin{gathered}
T_{S}^{m c}=R T S+S I F S+\delta+C T S_{m c}+S I F S+\delta+H+E[P]+S I F S+\delta+A C K_{m c}+D I F S+\delta \\
T_{C}^{b a s}=R T S+D I F S+\delta .
\end{gathered}
$$

If the number of receivers $(R)$ exceeds the codelength $\left(L_{C}\right)$, the CTS/ACK has to transmit more than twice. If $L_{C}<R$, equation (9) has to be modified

$$
T_{S}^{m c}=R T S+\left\lceil\frac{R}{L_{C}}\right\rceil\left(S I F S+\delta+C T S_{m c}\right)+S I F S+\delta+H+E[P]+\left\lceil\frac{R}{L_{C}}\right\rceil\left(S I F S+\delta+A C K_{m c}\right)+D I F S+\delta
$$

\subsection{Packet Delay Analysis}

We use an approach similar to [14] to derive the average delay of the successful packet transmissions. The average delay for a successfully transmitted packet is defined to be the time interval from the time the packet is at the head of its MAC queue ready to be transmitted, until an acknowledgement for this packet is received. If a packet is dropped, as it has reached the specified retry limit, the delay time for this packet will not be included in the calculation of the average delay. The average packet delay $E[D]$ for packets is given by

$$
E[D]=E[X] E[\text { slot }]
$$

where $E[$ slot $]$ is given by (6), and $E[X]$ is the average number of slot times required for successfully transmitting a packet and is given by

$$
\begin{aligned}
E[X] & =\sum_{i=o}^{m}\left(\frac{W_{i}+1}{2}\right) \cdot \sum_{k=0}^{W_{i}-1} b_{i} k=\sum_{i=0}^{m}\left(\frac{W_{i}+1}{2}\right) \cdot b_{i . o} \sum_{k=0}^{W_{i}-1}\left(\frac{W_{i}-k}{W_{i}}\right) \\
& =\sum_{i=o}^{m}\left(\frac{W_{i}+1}{2}\right) \cdot b_{i .0}\left(\frac{W i+1}{2}\right)=\sum_{i=o}^{m}\left(\frac{W_{i}+1}{2}\right)^{2} \cdot b_{i .0} \\
& =\sum_{i=o}^{m}\left\{\left(\frac{W_{i}+1}{2}\right)^{2} \cdot p_{i} \cdot b_{0.0}\right\}+\frac{b_{0.0}}{1-p} \cdot p^{m} \cdot\left(\frac{W \cdot 2^{m}+1}{2}\right)^{2} \\
& =\sum_{i=0}^{m-1}\left\{\tau \cdot(1-p) p^{i} \cdot\left(\frac{W_{i}+1}{2}\right)^{2}\right\}+\tau \cdot p^{m}\left(\frac{W_{m}+1}{2}\right)^{2} \\
& =\tau\left[\sum_{i=0}^{m-1}\left\{(1-p) p^{i} \cdot\left(\frac{W^{i}+1}{2}\right)^{2}\right\}+p^{m} \cdot\left(\frac{W_{m}+1}{2}\right)^{2}\right]
\end{aligned}
$$

where, $b_{i, k}$ value can be referred to [14]. 
International Journal of Computer Networks \& Communications (IJCNC) Vol.3, No.5, Sep 2011

\subsection{Calculation of the SRR}

We now compute the $S R R$ and multiply this by $S_{I}$ to obtain the total network throughput of a multiple multicast group. [15] derives the equation of transmission range $\left(d_{t r}\right)$ and carrier sensing range $\left(d_{c s r}\right)$ solely as a function of the transmission power $\left(P_{t}\right)$ under the condition that other parameters are fixed.

$$
\begin{aligned}
& d_{t r}=\sqrt[4]{\frac{P_{t} G_{t} G_{r} H_{t}{ }^{2} H_{r}{ }^{2}}{t h r d_{r x}}} \\
& d_{c s r}=\sqrt[4]{\frac{P_{t} G_{t} G_{r} H_{t}^{2} H_{r}^{2}}{t h r d_{c s}}}
\end{aligned}
$$

where $G_{t}$ and $G_{r}$ are the antenna gain of the transmitter and receiver, $H_{t}$ and $H_{r}$ denote the antenna height of the transmitter and receiver, $t_{h r} d_{r x}$ is the power level at which a receiver can detect and correctly decode the transmitted signal, $t h r d_{c s}$ is the power level at which a node can detect the transmitted signal with sufficient power to "sense to carrier". In a CSMA/CA MAC, all nodes within $d_{c s r}$ of an ongoing transmission should defer their own transmission until the channel is idle. $d_{c s r}$ defines the potential set of EN.

In (13) and (14), we can decrease $P_{T}$ as $\left(1 / L_{C}\right)$ by the processing gain of the proposed protocol [16]. Other parameters, excluding $P_{T}$, are fixed, when the signal is spread, $d_{t r}$ and $d_{c s r}$ decrease by $\left(1 / L_{C}\right)^{1 / 4} \cdot d_{t r}$ and $d_{c s r}$ decrease by $\left(1 / L_{C}\right)^{1 / 4}$, since [20] derives these equations from the twoway ground path-loss model.

Fig. 4 shows the network configuration for the simulation. Six neighbor multicast groups surround one multicast group. Only the center node (S1 S7) of each multicast group can be a sender. Each $d_{t r}$ does not overlap with each other, each $d_{c s r}$ does. The multicast receivers of each multicast group are randomly located in $d_{t r}$. The network can be extended, keeping the same configuration. We form the network, such as Fig. 4, since the influence of EN caused by CTS overhearing range is maximized with no influence caused by RTS.

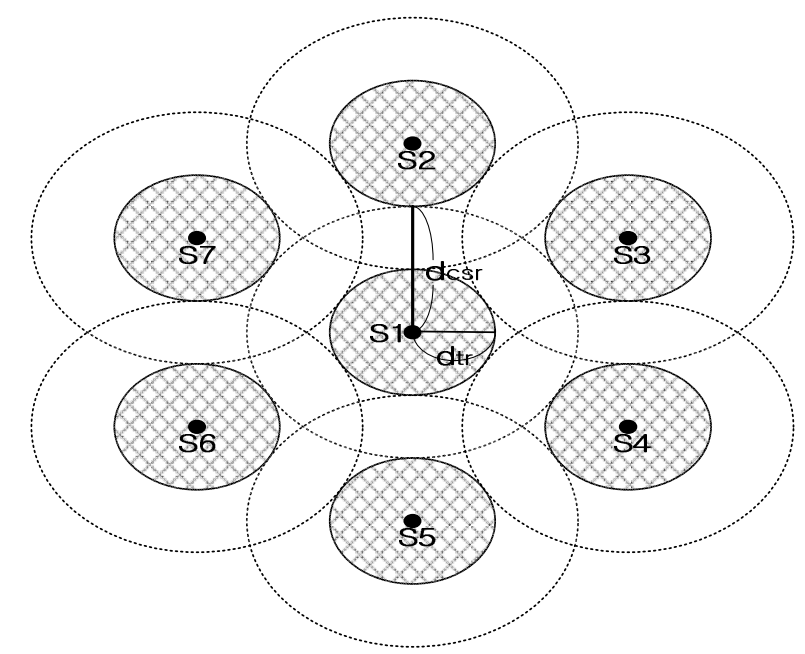

Figure 4. Network Configuration for the $S R R$ simulation. 
International Journal of Computer Networks \& Communications (IJCNC) Vol.3, No.5, Sep 2011

Each multicast group sender (S1 S7) has a packet to transmit in each transmission queue to be consistent with the saturation throughput model. If S1 transmits an mRTS message to the multicast receivers in its own $d_{t r}$, the randomly chosen receivers also have their own $d_{t r}$ and $d_{c s r}$ response to CTS. At this time, if at least one station among the multicast receivers of another neighbor multicast group is in $d_{c s r}$ of each multicast receiver of $\mathrm{S} 1$, that neighbor multicast group is exposed. Therefore the number of exposed multicast groups is counted within range of 1 to 6 . In our simulation, the number of stations $(n)$ in one multicast group is fixed at 50 and the number of multicast receivers is changed from 5 to 50 . Finally, the $S R R$ is calculated, as follows.

$$
S R R=\frac{\left(N_{G}+1\right)-\text { number of exposed multicast group }}{N_{G}+1}
$$

where, $N_{G}+1$ is a number of neighbor multicast groups, including itself.

Table 1. . Simulation parameter values.

\begin{tabular}{|c|c||c|c|}
\hline Parameter & Value & Parameter & Value \\
\hline CWmin & $15 \mu \mathrm{sec}$ & PHY header & $46 \mathrm{bits}$ \\
\hline CWmax & 1023 & Preamble & $16 \mu \mathrm{sec}$ \\
\hline RTS time & $52 \mu \mathrm{sec}$ & CTS/ACK time & $44 \mu \mathrm{sec}$ \\
\hline SIFS time & $16 \mu \mathrm{sec}$ & Data rate & $6 \mathrm{Mbps}$ \\
\hline DIFS time & $34 \mu \mathrm{sec}$ & Packet payload & $8192 \mathrm{bits}$ \\
\hline Slot time & $9 \mu \mathrm{sec}$ & $d_{\text {tr }}^{\text {bas }}$ & $400 \mathrm{~m}$ \\
\hline$\delta$ & $1 \mu \mathrm{sec}$ & $d_{c s r}^{\text {bas }}$ & $670 \mathrm{~m}$ \\
\hline MAC header & $272 \mathrm{bits}$ & & \\
\hline
\end{tabular}

\section{NUMERICAL RESULTS}

The analytical model and simulation results are implemented by a simulator to verify the performance of the proposed protocol. We use the MATLAB simulator. Table 1 summarizes the values of the parameters used to obtain the numerical results for the analytical model and the simulation runs. The values of these parameters are based on the IEEE 802.11a standard [17].

The proposed protocol is compared to the conventional protocol using multiple CTSs and ACKs, such as [5] and [6]. We only compare this basic multiple CTSs/ACKs protocol, since we are only interested in a reliable transmission, as explained in section 1. Among the protocols explained in section 2, the only protocol supporting a reliable service with RTS-CTS handshake is the MAC protocol using multiple CTSs/ACKs, such as [5] and [6]. 
International Journal of Computer Networks \& Communications (IJCNC) Vol.3, No.5, Sep 2011

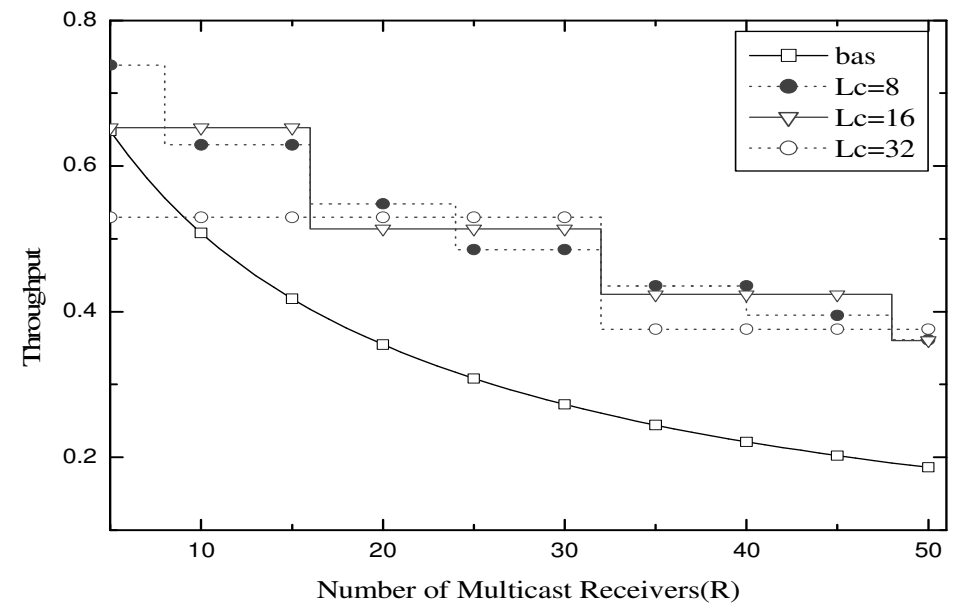

Figure 5. Number of multicast receivers and throughput disregarding EN

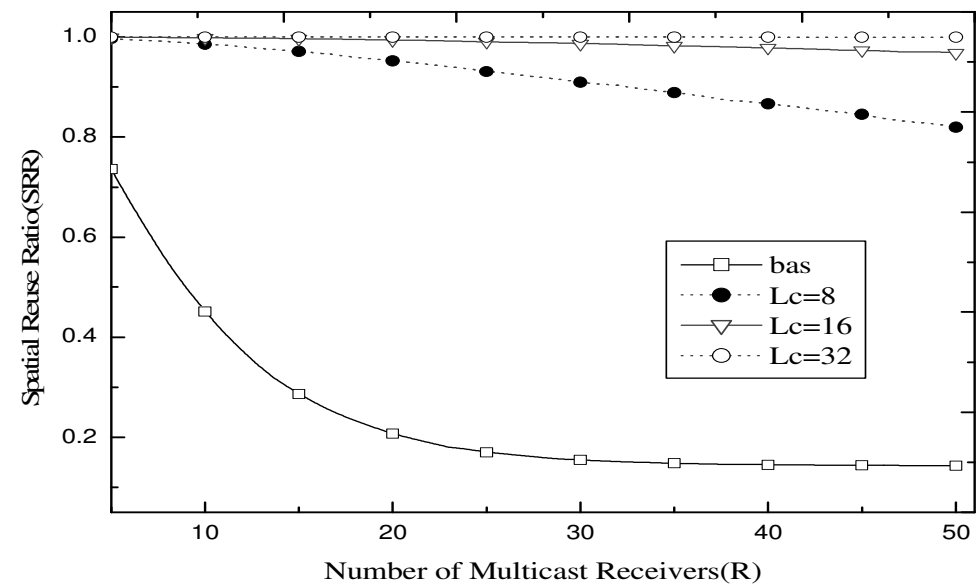

Figure 6. Spatial reuse Ratio (SRR)

Fig. 5 shows the variation of the throughput of the proposed MAC and the multiple CTS/ACKs MAC with the number of multicast receivers $(R)$, where $n=50$, the codelength is 8,16 and 32 , the $R$ is varied from 5 to 50 , not considering $S R R$ (regarding one multicast group in the network). Though we do not consider $S R R$, the performance of the proposed MAC is better than for the basic one. Furthermore, as the $R$ value is higher, the gain difference between the basic protocol and the proposed one gets bigger and bigger. The throughput of both decreases as the number of multicast receivers increases. The curve of the proposed scheme is bent at a point (the point that the number of multicast receivers exceeds the codelength) of multiple numbers of codelength, because the CTS/ACK is sent more than twice.

Fig. 6 shows the variation of the simulation results of the $S R R$ with $R$. The $S R R$ decreases severely when $R$ increases in a basic protocol. However, the proposed protocol performs much better as $R$ increases, since when the codelength is lengthened, $d_{t r}$ and $d_{c s r}$ decrease, and the EN area is degraded. The $S R R$ approaches 1 at codelengths of 16 and 32; this means that the neighbor multicast group can transmit each multicast packet concurrently. 


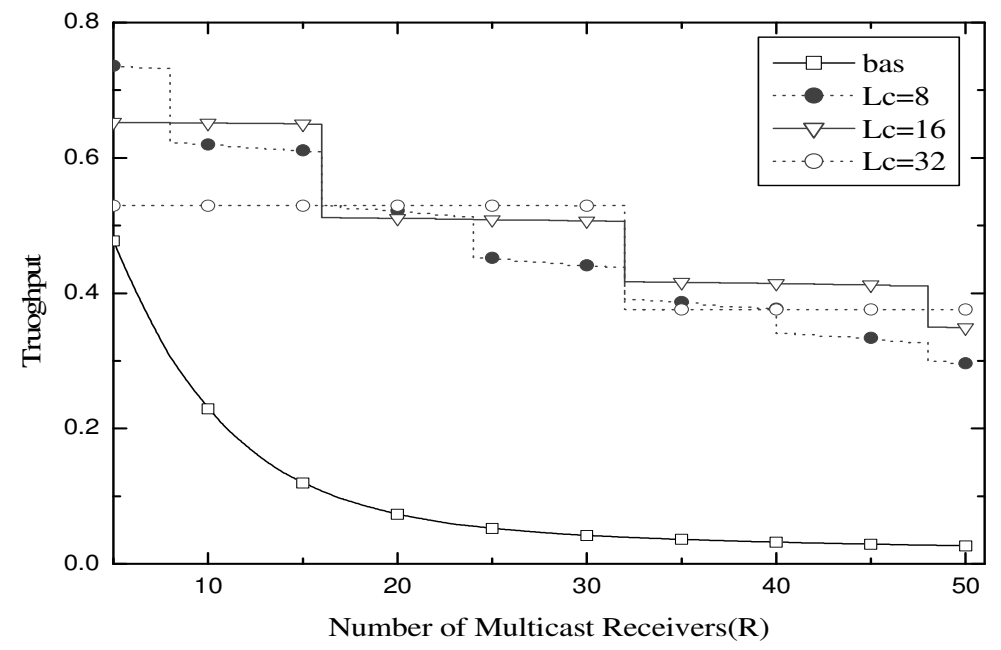

Figure 7. Number of multicast receivers and throughput regarding SRR

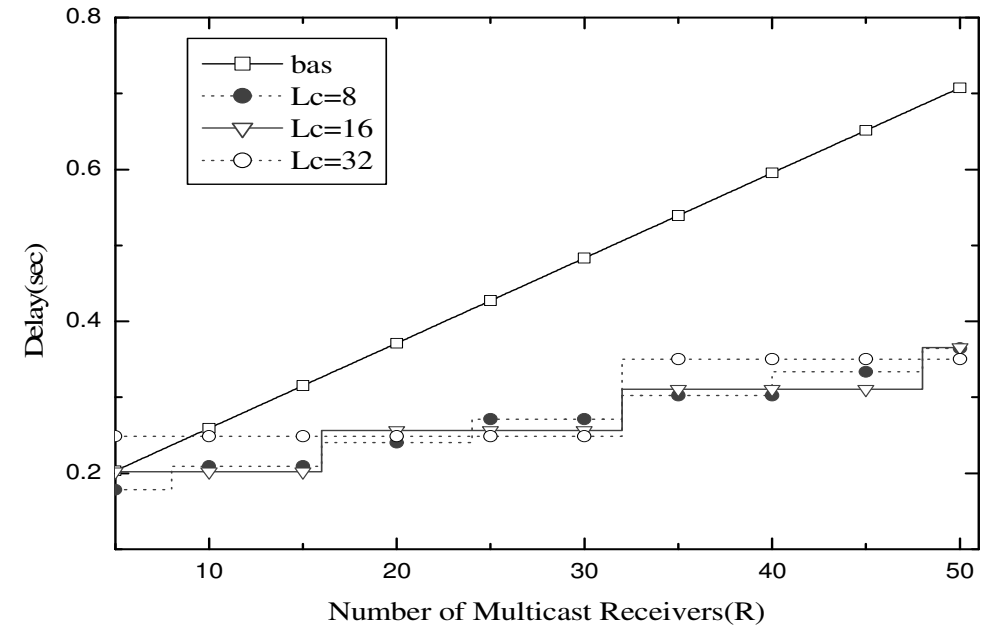

Figure 8. Number of multicast receivers and packet delay disregarding EN

Fig. 7 shows the total throughput, calculated by multiplying $S R R$ by the result of Fig. 5, of the network considering the effect of the $S R R$. In the result shown, the margin between the proposed MAC and basic one becomes larger than that in Fig. 5. The throughput of the basic one decreases drastically, but the proposed scheme experiences only minor change.

Fig. 8 shows the packet delay time, disregarding the effect of the $S R R$, calculated by equations (11) and (12), as $R$ increases from 5 to 50. Fig. 8 shows the delay time increases with increasing $R$. The curves of the proposed scheme are also bent at the point of the multiple number of the codelength. 
International Journal of Computer Networks \& Communications (IJCNC) Vol.3, No.5, Sep 2011

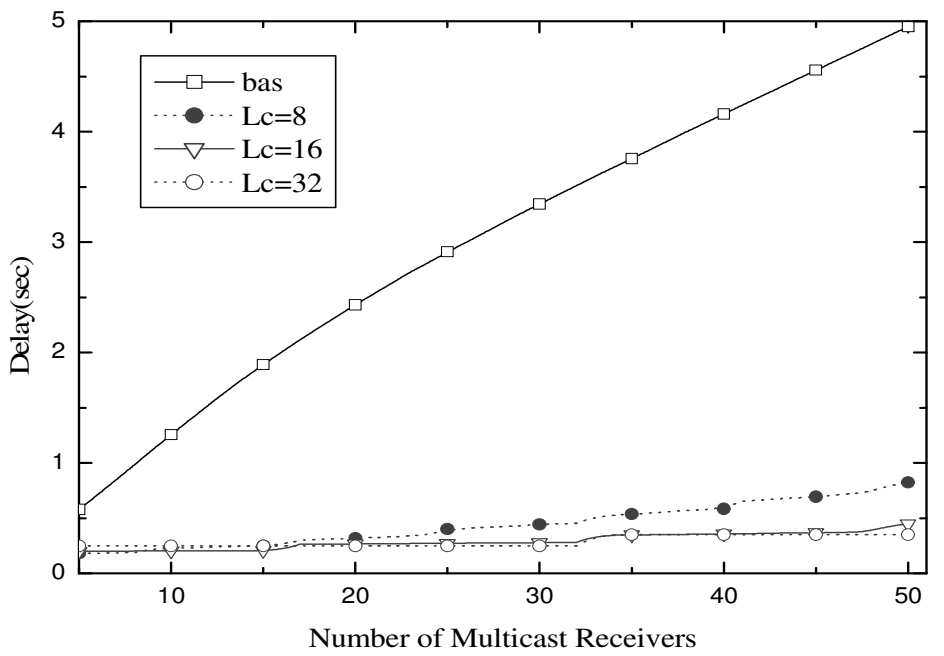

Figure 9. Number of multicast receivers and packet delay regarding EN

Fig. 9 shows the variations of the packet delay time considering SRR. This means the transmission deferral time of each multicast group by EN. We can calculate this by multiplying the inverse of the SRR. In Fig. 9, the delay time of the basic one also drastically increases, since the effect of EN delays the transmissions of neighbors.

\section{CONCLuSiON}

In this paper, we proposed a new reliable multicast MAC protocol over the IEEE 802.11-based WLAN. The major innovation over previous reliable multicast protocols using consecutive CTSs/ACKs is that this can send multiple CTS/ACK messages concurrently via the MC DSCDMA scheme. When multiple receivers receive a RTS/DATA message from a sender, they respond with a CTS/ACK message spread with the pre-assigned code in the same time interval. This can reduce the overhead of multiple CTSs/ACKs. It is possible to alleviate the EN area over decreasing transmission range and carrier sensing range of each receiver. Our proposed protocol and the multiple CTSs/ACKs based multicast protocol were compared and evaluated using an analytical method and simulation. The proposed protocol outperforms the conventional protocol based on multiple CTSs/ACKs in terms of throughput and delay. That is, our proposed protocol provides an efficient reliable multicast MAC mechanism with minimum overhead.

\section{ACKNOWLEDGEMENTS}

"This research was supported by MKE (The Ministry of Knowledge Economy), Korea, under the ITRC (Information Technology Research Center) support program supervised by the NIPA (National IT Industry Promotion Agency" (NIPA-2011-C1090-0902-0003)

\section{REFERENCES}

[1] C. Cordeiro, H. Gossain and D. Agrawal, "Multicast over Wireless Mobile Ad Hoc Networks: Present and Future Directions", in IEEE Network, SI on Multicasting: An Enabling Technology, January/February 2003 
International Journal of Computer Networks \& Communications (IJCNC) Vol.3, No.5, Sep 2011

[2] K. Obraczka, "Multicast transport protocols: A survey and taxonomy," IEEE Commun. Mag., vol. 36, no. 1, pp. 94-102, Jan. 1998.

[3] A. El-Sayed, V. Roca, and L. Mathy, “A survey of proposals for an alternative group communication service,” IEEE Network, vol. 17, no. 1, pp. 46-51, Jan./Feb. 2003.

[4] M. T. Sum, L. Huang, A. Arora, and T. H. Lai, "Reliable MAC layer multicast in IEEE 802.11 wireless networks,” Wireless Commun. Mob. Comput., vol. 3, no. 4, pp. 439-453, Jun. 2003.

[5] H. Gossain, N. Nandiraju, K. Anand, and D. P. Agrawal, "Supporting MAC layer multicast in IEEE 802.11 based MANET's: Issues and solutions," in Proc. IEEE LCN, pp. 172-179, Nov. 2004.

[6] Ki-Ho Lee and Dong-Ho Cho. "A Multiple access collision avoidance protocol for multicast service in mobile ad hoc networks," in Proc. Vehicular Technology Conference, 2003.

[7] J. Kuri and S. K. Kasera, "Reliable multicast in multi-access wireless LANs," Wireless Netw., vol. 7, no. 4, pp. 359-369, Jul. 2001.

[8] B. Kim, S. W. Kim, and R. L. Ekl, "OFDMA-based reliable multicasting MAC protocol for WLANs," IEEE Transactions on Vehicular Technology, vol. 57, no. 5, pp. 3136-3145, September 2008.

[9] Shukla, D.; Chandran-Wadia, L.; Iyer, S. "Mitigating the exposed node problem in IEEE 802.11 ad hoc networks,” In Proc. IEEE ICCCN'03, Dallas, TX, pp. 157-162, Oct. 2003.

[10] Haas, Z.; Deng, J. "Dual busy tone multiple access (DBTMA)-a multiple access control scheme for ad hoc networks,” IEEE Trans. Commun., pp.975-985, 2002.

[11] Acharya, A.; Misra, A.; Bansal, S. "MACA-P: A MAC for concurrent transmissions in multihop wireless networks," In Proc. IEEE Pervasive Computing and Communications (PerCom'03), Dallas-Fort Worth, TX, USA, pp. 505-508, Mar. 2003.

[12] S. Hara and R. Prasad, "Overview of multicarrier CDMA," IEEE Commun. Mag., Vol. 35, No. 12, pp. 126- 133, 1997.

[13] G. Bianchi, "Performance analysis of the IEEE 802.11 distributed coordination function," IEEE J. Select. Areas Commun., vol. 18 pp 535-547, Mar. 2000.

[14] P. Chatzimisios, A.C. Boucouvalas, and V. Vitsas, "IEEE 802.11 packet delay - a finite retry limit analysis,” in Proc. IEEE Globecom, San Francisco, USA, Dec. 2003.

[15] Zhou, Y.; Nettles, S. "Balancing the hidden and exposed node problems with power control in CSMA/CA-based Wireless Networks," In Proc. IEEE WCNC'05, New Orleans, LA, USA, pp. 683-688, Mar. 2005.

[16] Yang. S. C. CDMA RF System Engineering. Norwood, MA : Artech House. 1998

[17] Wireless LAN Medium Access Control (MAC) and Physical Layer (PHY) Specifications: Higher-Speed Physical Layer in the 5 GHz Band, IEEE Std. 802.11a-1999.

[18] S. Mehta and K.S. Kwak, “ Comparison of Difficult Broadcast Schemes for Multi-hop Wireless Sensor Networks”, IJCNC, Vol.2, No.4, July 2010

\section{Authors}

Jeonghun Kim received the B.S. in international relations from Naval Academy, Korea, in 2000. Since March 2008, he has been pursuing his Ph.D, in Ajou University. His research interests are related to MAC protocol, resource management and multiple access for the wireless communications systems.

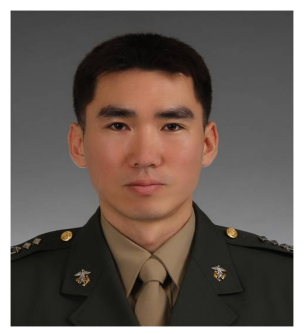


International Journal of Computer Networks \& Communications (IJCNC) Vol.3, No.5, Sep 2011

Junwoo Jung received the B.S. in information and computer engineering and the M.S. in information and communication engineering from Ajou University, Korea, in 2005 and 2007, respectively. Since March 2007, he has been pursuing his Ph.D, in Ajou University. His research interests are related to cooperative communication, MAC protocol, resource management and multiple access for the wireless communications systems.

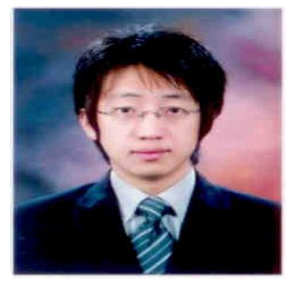

Jaesung Lim received the B.S. in electronic engineering from Ajou University, Korea, in 1983, and the M.S. and Ph.D, in electrical engineering from Korea Advanced Institute of Science and Technology (KAIST), in 1985 and 1994, respectively. In 1985, he started as a reseacher at the DAEWOO telecommunication. In April 1988, he joined the institute of DigiCom, and was engaged in research and development of data modem, radar signal processing and packet data systems. From 1995 to 1997, he served as a senior engineer in the Central Research \& Development Center of SK Telecom, where he did

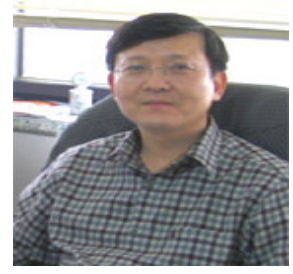
research on wireless data communications for cellular and paging networks. Since March 1998 he has been with Ajou University, where he is a professor of the Graduate School of Information and Communication Technology, teaching and doing research in the areas of wireless/mobile systems and tactical networks. 